\title{
Congenital cardiovascular defects in children with intestinal malrotation
}

\author{
M. Kouwenberg · R. S. V. M. Severijnen • \\ L. Kapusta
}

Accepted: 8 November 2007/Published online: 11 December 2007

(C) The Author(s) 2007

\begin{abstract}
Intestinal malrotation (IM) and cardiovascular defects (CCVD) are both common congenital defects. We investigated the prevalence and types of CCVD in a 25year IM population, and its association with post-IMoperative morbidity and mortality. Data on the type of CCVD, other congenital defects, syndromes, associations, post-IM-operative morbidity and mortality were retrospectively reviewed from the records of IM patients born between 1980 and 2005. Data were analyzed on (significant) differences between CCVD subgroups, and risk factors for both morbidity and mortality were calculated. Seventy-seven of 284 IM patients $(27.1 \%)$ were diagnosed with a major or minor CCVD (37 and 40 patients, respectively). Syndromes and associations were more frequently diagnosed in patients with major than with a minor CCVD (67.6 vs. $40 \%$, respectively). Post-IM-operative complications, although frequently observed (61\%), did not differ between patients with major and minor CCVD. Physical CCVD signs before IM surgery increased postIM-operative morbidity significantly (OR 4.0, 95\% CI 1.411.0). Fifteen patients died (19.5\%), seven due to
\end{abstract}

\footnotetext{
M. Kouwenberg

Department of Paediatrics, Radboud University Nijmegen

Medical Centre, Nijmegen, The Netherlands

e-mail: m.kouwenberg@cukz.umcn.nl

R. S. V. M. Severijnen

Department of Paediatric Surgery, Radboud University

Nijmegen Medical Centre, Nijmegen, The Netherlands

e-mail: r.severijnen@kchir.umcn.nl

L. Kapusta $(\bowtie)$

Children's Heart Center, Radboud University Nijmegen Medical

Centre, P.O. Box 9101, 6500 HB Nijmegen, The Netherlands

e-mail: 1.kapusta@cukz.umcn.nl
}

cardiovascular cause. Mortality risk was increased by intestinal ischemia and post-IM-operative complications and by major CCVD after correction for age at weight at the time of IM operation. Congenital cardiovascular defects in children with intestinal malrotation are common, with high morbidity and mortality rates after IM operation. Elective IM surgery in young patients with CCVD should be performed in a centre with adequate paediatric cardiac care. Benefits of laparoscopic intervention need further study.

Keywords Intestinal malrotation .

Congenital cardiovascular defect .

Post-operative morbidity · Post-operative mortality

\section{Introduction}

Intestinal malrotation (IM) is refers to all abnormalities of intestinal position and attachment and includes the concept of atypical malrotation or malrotation variant [1]. It is often accompanied by a narrow base of the small bowel mesentery. The incidence of IM is 1:500-1:3,500 living births [2, $3]$. Volvulus, a rotation of the gut along its mesenteric stalk occurs in $60-70 \%$ of neonates diagnosed with IM, the ultimate strangulation in about $15 \%$ [4]. Eighty percent of the patients with symptomatic IM present in their first month of life [5] and require surgery. Delay in diagnosis may lead to intestinal ischemia, intestinal necrosis, septicaemia and short bowel syndrome. The surgical outcome is negatively influenced by the presence of intestinal necrosis [6], and the need for operation during the first 3 months of life [6-9]. To keep mortality and morbidity low, all asymptomatic children with intestinal malrotation are advised to undergo semi-elective surgery (Ladd's procedure) in childhood and adolescence [10,11]. 
Intestinal malrotation has been described in almost 100 syndromes and combinations. Associated serious abnormalities increase the mortality risk in patients with IM of about 22-fold according to Messineo [6]. Commonly associated congenital malformations are duodenal atresia, abdominal wall defects or diaphragmatic hernia $[3,12]$. It is more frequently observed in patients with situs inversus [13] or heterotaxia, a syndrome associated by congenital cardiovascular defects [14].

Congenital cardiovascular defects (CCVD) are among the most common congenital birth defects occurring in about 1 in 100 living births [15]. Congenital malformations of the gastrointestinal, musculoskeletal and urinary systems are the most frequently seen extra cardiac malformations in patients with CCVD [16]. Intestinal malrotation has been observed in $2.8-4.1 \%$ of all the patients with CCVD, mainly in patients with single ventricle [17]. However, the incidence of CCVD in IM patients has not been precisely quantified in the recent literature and the full array of the associated cardiac anomalies has not been well described.

The purpose of our study was (1) to delineate and accurately describe the types and incidence of associated cardiac lesions in our most recent 25-year experience of patients with IM, and (2) to study the influence of a coexisting CCVDs on IM surgery morbidity and mortality.

\section{Materials and methods}

Population

A retrospective review at a single university centre was conducted on all the paediatric patients diagnosed with IM born and between September 1980 and September 2005. Patients with CCVD were identified for further analysis. Patients demographics recorded included gestational age, weight and gender. Data on the type of CCVD, other congenital defects, known syndromes or associations, postoperative complications, morbidity and mortality were collected. The diagnosis of CCVD was based on clinical, ECG, echocardiography, heart catheterization and perioperative findings. The IM diagnosis was based on radiological upper gastro-intestinal examination, or during acute surgery.

\section{Data analysis}

Collected data were analyzed by calculating prevalence percentages. Analysis of nominal variables was performed using cross tables for calculating relative risk (RR) with the corresponding 95\% confidence interval (95\% CI). Fisher extact test was used for comparison of percentages of both
CCVD groups, expressed as $P$ values. For the comparison of continuous variables, Mann-Whitney $U$ test was used to calculate $P$ value. The $P$ values are two-sided, and a value of $P=0.05$ was considered statistical significant. For the calculation of risk factors for both post-operative morbidity and mortality, (adjusted) odds ratios (OR) with consequent 95\% CI were calculated by uni- and multi-variate regression analysis. Statistical analysis was performed using SPSS software (version 12.0.1; SPSS Inc., Chicago, IL, USA).

\section{Results}

Demographics

During a period of 25 years, 284 patients were diagnosed in our centre with IM. Ninety-three of the 284 patients were identified with CCVD as well. Fifteen patients had persistent pulmonary hypertension (PPHT), all but one with congenital diaphragmatic hernia. One patient had a solitary pre-duodenal portal vein. The records of these 16 patients without structural CCVD were excluded, leaving 77 patients with both IM and CCVD (27.1\% of the total IM population in the last 25 years) for further analysis. The gestational age range was 26-42 weeks with a median of 39 weeks. Median birth weight was $2.86 \mathrm{~kg}$ (range $0.72-$ 4.53). A female predominance was seen with 47 girls $(61 \%)$ and 30 boys (39\%). Median follow-up period after IM diagnosis was 2.8 years (range 0-24 years). The overall survival at the end of follow-up was $80.5 \%$ (15 out of 77 patients died).

\section{Congenital cardiovascular defects}

The CCVDs were divided into the subgroups, major and minor CCVDs (see Table 1), varying from small ventricular septal defect (VSD) to common arterial trunk (CAT). Thirty-seven out of 77 patients $(48.1 \%)$ were diagnosed with major CCVD and 40 patients $(51.9 \%)$ with minor CCVD.

The characteristics of the whole group $(n=77)$ as well as that of the subgroups are described in Table 2. Forty-two patients underwent cardiac surgery, of which 20 patients had two or more cardiac surgical interventions. Patients with major CCVDs needed cardiac surgery more often. Minor CCVDs that needed surgical intervention most frequent were VSD, atrial septal defects (ASD) and aortic valve stenosis (AS). Six patients with severe CCVD did not undergo correction of their heart disease before IM operation: three patients died ( $n=2$ due to post IM surgery complications, $n=1$ because of other congenital malformations). One patient with dextrocardia did not need 
Table 1 Types of major and minor congenital cardiovascular defects

CCVD Congenital cardiovascular defects

\begin{tabular}{llll}
\hline Major CCVD & $n=37(100 \%)$ & Minor CCVD & $n=40(100 \%)$ \\
\hline $\begin{array}{l}\text { Complex cardiovascular } \\
\text { defects }\end{array}$ & $n=18(48.6 \%)$ & Ventricular septal defect & $n=16(40.0 \%)$ \\
$\begin{array}{l}\text { Tetralogy of Fallot } \\
\begin{array}{l}\text { Hypoplastic left heart } \\
\text { syndrome }\end{array}\end{array}$ & $n=7(18.9 \%)$ & Atrium septal defect & $n=7(17.5 \%)$ \\
$\begin{array}{l}\text { Atrioventricular septal defect } \\
\text { Pulmonary valve atresia }\end{array}$ & $n=3(8.1 \%)$ & Persistent ductus arteriosus & $n=3(10.0 \%)$ \\
$\begin{array}{l}\text { Transposition of the great } \\
\text { arteries }\end{array}$ & $n=1(2.7 \%)$ & Pulmonary valve stenosis & $n=3(7.5 \%)$ \\
$\begin{array}{l}\text { Tricuspid valve atresia } \\
\text { Common arterial trunk }\end{array}$ & $n=1(2.7 \%)$ & Aortic coarctation & $n=2(5.0 \%)$ \\
Aortic arch hypoplasia & $n=1(2.7 \%)$ & Hypertrophic cardiomyopathy & $n=1(2.5 \%)$ \\
Dextrocardia & $n=1(2.7 \%)$ & Subvalvular aortic valve stenosis & $n=1(2.5 \%)$ \\
& $n=1(2.7 \%)$ & Left superior caval vein & $n=1(2.5 \%)$ \\
& & Arteria lusoria & $n=1(2.5 \%)$ \\
\hline
\end{tabular}

Table 2 Characteristics of studied population and its subgroups of congenital cardiovascular defects

\begin{tabular}{lllll}
\hline Characteristic & $\begin{array}{l}\text { All CCVD } \\
100 \%(n=77)\end{array}$ & $\begin{array}{l}\text { Major CCVD } \\
100 \%(n=37)\end{array}$ & $\begin{array}{l}\text { Minor CCVD } \\
100 \%(n=40)\end{array}$ & Relative risk (95\% CI)/P value \\
\hline Boys & $39 \%(n=30)$ & $35.1 \%(n=13)$ & $42.5 \%(n=17)$ & $0.85(0.5-1.4)$ \\
Syndromes or associations & $53.2 \%(n=41)$ & $67.6 \%(n=25)$ & $40 \%(n=16)$ & $\mathbf{1 . 8}(\mathbf{1 . 1}-\mathbf{3 . 1})$ \\
Other congenital defects & $84.4 \%(n=65)$ & $83.8 \%(n=31)$ & $85 \%(n=34)$ & $0.95(0.5-1.8)$ \\
Cardiac surgery & $54.4 \%(n=42)$ & $83.8 \%(n=31)$ & $27.5 \%(n=11)$ & $\mathbf{4 . 3}(\mathbf{2 . 0 - 9 . 1})$ \\
CCVD diagnosed before IM & $57.1 \%(n=44)$ & $78.4 \%(n=29)$ & $37.5 \%(n=15)$ & $\mathbf{2 . 7}(\mathbf{1 . 4 - 5 . 2})$ \\
Age at CCVD diagnosis in days [median (range)] & $4.5(0-210)$ & $4(0-98)$ & $7(0-210)$ & $P=0.12$ \\
Birth weight in kilograms [median (range)] & $2.86(0.72-4.53)$ & $2.96(0.96-4.53)$ & $2.84(0.72-4.30)$ & $P=0.4$ \\
Age at first CCVD surgery in days [median (range)] & $102(2-3,759)$ & $43(2-3,759)$ & $117(24-1,705)$ & $P=0.15$
\end{tabular}

Values in bold represent statistical significant results

$C C V D$ Congenital cardiovascular defects, IM Intestinal malrotation

cardiac correction. The two patients with Tetralogy of Fallot (TOF) underwent cardiac correction 6 months after IM surgery.

Congenital malformations, syndromes and associations

Congenital malformations other than IM and CCVD were observed in 65 of the $77(84.4 \%)$ patients, see also Table 2. Most frequently observed congenital malformations included omphalocele $(n=7)$, congenital diaphragmatic hernia $(n=7)$, ano-rectal malformations $(n=6)$ and heterotaxia $(n=6)$.

Syndromes and associations were recognized in 41 $(53.2 \%)$ patients, of which Down syndrome $(n=7)$, heterotaxia $(n=6)$, VACTERL $(n=5)$, Ivemark $(n=5)$ and lateralization abnormalities $(n=4)$ were most frequently recognized. Kartagener syndrome occurred in two patients. Several other syndromes all occurred once. Syndromes and associations were more frequent in patients with major CCVD.
Intestinal malrotation surgery

Characteristics of IM-operated patients and their major and minor CCVD subgroups are described in Table 3. Five patients did not undergo IM surgery yet; in three patients the surgery was about to take place in the near future, in one patient no surgical intervention was planned and one patient died of an unknown cause before IM surgery could take place. A Ladd procedure was performed for IM correction. Twenty-eight out of the 72 operated patients needed acute IM surgery, at a median age of 4.0 days (range $0-455$ ). Only 4 out of these 28 patients had previously undergone surgical correction of the CCVD. Five patients had ischemic bowel disease at time of acute IM intervention; three of these patients died, one due to massive intestinal necrosis (one patient died due to cardiovascular cause, one patient died because of severe infection). At the time of IM surgery, signs and symptoms of CCVD, e.g., hepatomegaly, central cyanosis, cardiac murmurs and respiratory insufficiency, were more often observed twice in patients with a major CCVD (62.2 vs. 
Table 3 Characteristics of general population, major and minor congenital cardiovascular defects groups operated on intestinal malrotation

\begin{tabular}{lllll}
\hline Characteristic & All CCVD & Major CCVD & Minor CCVD & $P$ value \\
\hline IM surgery & $100 \%(n=72)$ & $100 \%(n=34)$ & $100 \%(n=38)$ & - \\
Physical signs CCVD prior to IM surgery & $48.6 \%(n=35)$ & $67.6 \%(n=23)$ & $30.0 \%(n=12)$ & $\mathbf{0 . 0 0 4}$ \\
Post-IM-operative complications & $61.1 \%(n=44)$ & $67.6 \%(n=23)$ & $55.3 \%(n=21)$ & 0.3 \\
Post-IM-operative cardiovascular complications & $18.1 \%(n=13)$ & $20.6 \%(n=7)$ & $15.8 \%(n=6)$ & 0.8 \\
Relaparotomy & $18.1 \%(n=13)$ & $17.6 \%(n=6)$ & $18.4 \%(n=7)$ & 1.0 \\
Age at IM surgery in days [median (range)] & $17(0-1,293)$ & $104(0-1,196)$ & $6(0-1,293)$ & $\mathbf{0 . 0 0 6}$ \\
Weight at IM surgery in kilograms [median (range)] & $3.21(1.20-11.4)$ & $4.84(1.20-11.4)$ & $33(1.24-10.6)$ & $\mathbf{0 . 0 2}$ \\
Death in days after IM surgery [median (range)] & $28(0-1,814)$ & $33-1,814)$ & $39)$ & $8(3-60)$
\end{tabular}

Values in bold represent statistical significant results

CCVD Congenital cardiovascular defects, IM Intestinal malrotation

$30.0 \%$, respectively, $P=0.004)$. Most common complications were intestinal (Platzbauch, leakage of the bowel anastomosis resulting in sepsis, feeding difficulties with long-term necessity of nasogastrical tube feeding), infections and cardiovascular complications (e.g. bradycardia, thrombosis, arrhythmia) and they did not differ between both subgroups. Thirteen patients needed relaparotomy because of leakage of the bowel anastomosis $(n=4)$, Platzbauch $(n=2)$, suspected bowel obstruction with feeding difficulties $(n=1)$ and necrotising enterocolitis $(n=1)$. Five patients were reoperated for an adhesion ileus, all but one more than 1 month after surgery.

Patients suffering from a post-operative IM surgery complication were younger (5.5 vs. 94.5 days, $P=0.031$ ) and had a lower body weight (2.86 vs. $4.84 \mathrm{~kg}, P=0.003$ ) at time of IM surgery. Major CCVD, co-occurrence of a syndrome, or other congenital malformations did not significantly increase the risk on post-IM-operative morbidity. Correction for age and weight at the time of IM surgery in both CCVD groups showed, although not significant, a clear trend in post-IM-operative morbidity risk in patients diagnosed major CCVD (OR 2.9, 95\% CI 0.9-9.6). Patients with pre-operative signs of CCVD had a four times increased risk on post-operative complications (95\% CI 1.4-11.0). This risk factor was more prominent after correction for age and weight at the time of IM surgery (OR 8.2, 95\% CI 2.1-30.7).

\section{Mortality}

Fifteen (19.5\%) out of 77 patients died, 9 with major CCVDs. One patient with a minor CCVD died prior to IM surgery at the age of 1.4 years; cause of death remained unclear. Known causes of death after IM surgery were: cardiovascular complications $(n=7)$, massive intestinal necrosis $(n=2)$, sepsis $(n=2)$, respiratory failure $(n=2)$ and abstention of medical care because of unsuccessful surgical intervention on malformed trachea $(n=1)$. In both CCVD groups, death due to a cardiovascular cause remained the most frequent one. Median age at time of death was 40.5 days (range 3-3,010), with a median of 28.5 days after IM surgery (range 0-1,814). Of the 14 deceased patients, $9(64.3 \%)$ had a major CCVD. Median age at time of death in the major CCVD group was 46 days (range 22-3,010) and in the minor CCVD group 8 days (range 3-60 days), although this difference was statistically not significant $(P=0.4)$. Twenty percent of the patients (7/35) with pre-operative physical signs of CCVD died, only one due to a cardiovascular cause. Three out of five patients with ischemic bowel at the time of IM surgery died post-operatively. The risk factors for mortality are shown in Table 4.

\section{Discussion}

This study addresses the co-existence of malrotation with different types of congenital cardiovascular defects. We found $27.1 \%$ of all the patients registered in the last 25 years for IM to have a major or minor CCVD. Major CCVDs were seen in almost half of our population. Stewart et al. [2] found only $12 \%$ in a smaller IM population to have any CCVD. Complex CCVDs (23\% of our population, $6.3 \%$ of all patients with IM in the last 25 years) and VSDs (21\% of our population, $5.6 \%$ of all patients with IM in the last 25 years) were most frequently diagnosed. Pradat et al. [17] reported the co-occurrence of IM in patients with single ventricle to be as high as $8.5 \%$. Further data on co-occurrence of CCVD in IM patients are missing.

The high prevalence of the CCVD in our IM cohort needs some thoughts on common aetiology. The gut is developed at a later stage in embryogenesis than the heart does. Abnormal abdominal development, e.g. by early 
Table 4 Risk factors (expressed as odds ratios and 95\% confidence interval) for mortality in the whole study population $(n=77)$

Values in bold represent statistical significant results CCVD Congenital cardiovascular defects, $I M$ Intestinal malrotation

\begin{tabular}{|c|c|c|c|c|}
\hline Characteristic & Deceased & Alive & $\begin{array}{l}\text { Odds ratio } \\
(95 \% \mathrm{CI})\end{array}$ & $\begin{array}{l}\text { Adjusting for weight } \\
\text { and age at time of } \\
\text { IM surgery OR }(95 \% \mathrm{CI})\end{array}$ \\
\hline Major CCVD & 9 & 28 & \multirow[t]{2}{*}{$1.8(0.6-5.7)$} & \multirow{2}{*}{$5.0(1.2-20.7)$} \\
\hline Minor CCVD & 6 & 34 & & \\
\hline Syndrome/association & 7 & 34 & \multirow[t]{2}{*}{$0.7(0.2-2.2)$} & \multirow[t]{2}{*}{$1.0(0.3-3.4)$} \\
\hline No syndrome or association & 8 & 28 & & \\
\hline Other congenital defects & 13 & 52 & \multirow[t]{2}{*}{$1.3(0.2-6.4)$} & \multirow[t]{2}{*}{$1.1(0.2-5.9)$} \\
\hline No other congenital defects & 2 & 10 & & \\
\hline $\begin{array}{l}\text { Physical CCVD signs prior to } \\
\text { IM-surgery }\end{array}$ & 7 & 28 & \multirow[t]{2}{*}{$1.1(0.3-3.4)$} & \multirow[t]{2}{*}{$1.3(0.4-4.4)$} \\
\hline $\begin{array}{l}\text { No physical CCVD signs prior to } \\
\text { IM-surgery }\end{array}$ & 7 & 30 & & \\
\hline Intestinal ischemia & 3 & 2 & \multirow{2}{*}{$7.6(1.1-51.2)$} & \multirow[t]{2}{*}{$5.9(0.9-42.4)$} \\
\hline No intestinal ischemia & 11 & 56 & & \\
\hline Post-IM-operative complications & 1 & 27 & \multirow{2}{*}{$11.3(1.4-92.3)$} & \multirow{2}{*}{$8.7(1.0-75.7)$} \\
\hline $\begin{array}{l}\text { No post-IM-operative } \\
\text { complications }\end{array}$ & 13 & 31 & & \\
\hline $\begin{array}{l}\text { Post-IM operative cardiovascular } \\
\text { complications }\end{array}$ & 7 & 6 & \multirow[t]{2}{*}{$5.5(1.5-20.5)$} & \multirow[t]{2}{*}{$4.5(1.1-18.4)$} \\
\hline $\begin{array}{l}\text { No post-IM-operative } \\
\text { cardiovascular complications }\end{array}$ & 8 & 51 & & \\
\hline
\end{tabular}

development of an intra-abdominal cyst in embryogenesis, is known to enhance the chance of developing IM [18]. Changes in intra-abdominal space caused by a CCVD might also increase the risk on IM development during embryogenesis [4]. Another explanation might be a common genetic cause. The gut and the heart are both organs that show a characteristic left-right asymmetry, caused by a specific biochemical cascade during embryogenesis [19]. Two signalling molecules inevitable for this cardiac and gastrointestinal asymmetry are nodal and pitx2c [20]. Reduced, diffuse or absent expression of pitx $2 c$ in the embryo cause abnormal looping from both heart and gut in animal studies [21-24], resulting mainly in abnormal outflow tract orientation (e.g. unseptated and misaligned great artery trunks, symmetrical outflow tract cushions, double outlet right ventricle, common atrioventricular trunk) [23, 24]. This disturbed expression of pitx2c could cause a spectrum of isomerism [25]. More research is needed to reveal a possible common genetic cause for both CCVD and IM.

Our study group explores a high percentage of syndromes or associations. Although IM has been recognized in many syndromes and associations, this high prevalence should make us aware of co-occurrence of CCVD in these patients, as well as the possibility of a syndrome or association in patients diagnosed with both IM and CCVD. The post-operative morbidity after IM surgery in the general paediatric population is between 0 and $16.7 \%[9,26]$. A higher morbidity rate $(47 \%)$ has been reported by Murphy et al. [27], yet the surgical intervention was acute in $65 \%$ of their study population. Consistent with earlier reports [68], we found post-operative morbidity and mortality to be associated with younger age at the time of surgery. However, ischemic bowel (at the time of IM surgery) did not increase post-IM-operative morbidity as reported by others [2, 6, 8, 28]. Re-laparotomy in our population was consistent with earlier reports in the general population [26, 27]. The current study showed that patients with a CCVD had a relatively high morbidity rate after IM surgery $(61.1 \%)$. Syndromes, associations or the presence of other congenital malformations were not found to be risk factors for post IM morbidity. Cardiovascular complications were seen in $18.1 \%$ of our population. There are neither data on prevalence of cardiovascular complications after IM surgery in the general paediatric population, nor on influence of physical condition prior to IM surgery on the morbidity after the operation. Although the type of CCVD was not significantly associated with an increased risk on morbidity, there was a clear trend (towards increased risk on post-operative morbidity) in patients with major CCVD. Furthermore, patients with pre-operative physical signs of CCVD had a high post-operative morbidity rate $(77 \%)$.

The overall mortality rate in literature after IM surgery varies between 3 and 9\% [6, 27-29]. Ilçe et al. [8] found a mortality rate varying between 0 and $36 \%$ in patients mainly younger than 1 year, having almost all emergency intervention. A recent study of Murphy et al. [27] reported only 1 post-operative death in 46 patients due to cardiovascular consequences in a patient with congenital cyanotic 
heart disease. In the current study, the mortality rate was higher: almost one-fifth of the patients died. Patients who died were significantly younger at the time of IM surgery. Other risk factors for death were major CCVD (after correction for age and weight at the time of IM surgery), ischemic bowel at the time of IM surgery, post-IM-operative complications in general and post-IM-operative cardiovascular complications.

Diagnosis and treatment of cardiac diseases have incredibly improved in the last two decades. Associated congenital abnormalities, including IM, have increased in significance. However, since mortality rates after surgical intervention for IM in patients with an associated CCVD are high, intervention should be well thought over. Recent studies discuss the advantages of laparoscopic inspection for IM, also in acute paediatric abdomen [30]. No data on laparoscopy in IM patients with CCVD are available. Possible advantages of laparoscopy for IM diagnosis, followed by operation, even in acute cases, might improve the outcome [30], even in patients with major CCVD. Referral to a centre equipped with paediatric cardioanesthesiological care is imperative.

\section{Study limitations}

This is a retrospective study of all consecutive live-born IM patients referred to a single university centre over a 25 -year period. There are asymptomatic IM patients who never consult a doctor. Patients with IM and a very mild CCVD might have been missed. Screening for CCVD in IM patients could give us more information on the exact prevalence of CCVD in IM patients. Furthermore, since CCVD are very common congenital defects, a prospective study on the prevalence of IM in all patients with minor and major CCVD would also be of great interest.

Each retrospective study has its limitations, e.g. some variables were not (yet) available; e.g. syndromes and associations were common in our study population. Yet, the actual prevalence of syndromes might have been higher than reported. Certain syndromes and associations are quite new and nowadays there might be more attention on searching for a fitting syndrome or association diagnosis than 20 years ago.

As regards treatment, we perform 3 years laparoscopic inspection, followed by correction of the anomaly by laparoscopy or laparotomy in very young children [31].

\section{Conclusion}

The prevalence of CCVD in patients with IM is high (27.1\%). Major and minor CCVDs are both frequently seen. Major CCVD is a risk factor for death after IM operation. Since morbidity and mortality rates in IM patients with CCVD are much higher than previously reported, a pre-operative screening for co-existing CCVD is indicated. Elective IM surgery in young patients with major CCVD should be conducted in a centre with adequate paediatric cardiac care. The benefit of laparoscopic intervention needs further follow-up.

Acknowledgments The authors would like to thank Dr. P. Rieu for his contribution and comments on the manuscript, and Dr. T. Feuth for statistical support.

Open Access This article is distributed under the terms of the Creative Commons Attribution Noncommercial License which permits any noncommercial use, distribution, and reproduction in any medium, provided the original author(s) and source are credited.

\section{References}

1. Smith SD (2006) Disorders of intestinal rotation and fixation. In: Grosfeld JL, O’Neill JR (eds) Pediatric surgery. Elsevier, New York, p 1346

2. Stewart DR, Colodny AL, Willard CD (1976) Malrotation of the bowel in infants and children: a 15 year review. Surgery 79:716720

3. Forrester MB, Merz RD (2003) Epidemiology of intestinal malrotation, Hawaii, 1986-99. Paediatr Perinat Epidemiol 17:195200

4. Torres AM, Ziegler MM (1993) Malrotation of the intestines. World J Surg 17:326-331

5. Millar AJW, Cywes S (2003) Malrotation and volvulus in infancy and childhood. Semin Pediatr Surg 12:229-236

6. Messineo A, MacMillan JH, Palder SB, et al (1992) Clinical factors affecting mortality in children with malrotation of the intestine. J Pediatr Surg 10:1343-1345

7. Prasil P, Flageole H, Shaw KS, et al (2000) Should malrotation in children be treated differently according to age? J Pediatr Surg 35:756-758

8. Ilçe Z, CelayIr S, Akova F, et al (2003) Intestinal rotation anomalies in childhood: review of 22 years' experience. Surg Today 33:893-895

9. Powell DM, Othersen HB, Smith CD (1989) Malrotation of the intestines in children: the effect of age on presentation and therapy. J Pediatr Surg 24:777-780

10. Cohen Z, Kleiner O, Finaly R, et al (2003) How much of a misnomer is "asymptotic" intestinal malrotation? Isr Med Assoc J 5:172-174

11. Malek M, Burd RS (2006) The optimal management of malrotation diagnosed after infancy: a decision analysis. Am J Surg 191:45-51

12. Spigland N, Brandt M, Yazbeck S (1990) Malrotation presenting beyond the neonatal period. J Pediatr Surg 25:1139-1142

13. Lee SE, Kim H-Y, Jung S-E, et al (2006) Situs anomalies and gastrointestinal abnormalities. J Pediatr Surg 41:12371242

14. Choi M, Borenstein SH, Hornberger L, et al (2005) Heterotaxia syndrome: the role for screening for intestinal rotation abnormalities. Arch Dis Child 90:813-815

15. Hoffmann JI, Kaplan S (2002) The incidence of congenital heart disease. J Am Coll Cardiol 39:1890-1900 
16. Calzolari E, Garani G, Cocchi G, et al (2003) Congenital heart defects: 15 years of experience of the Emilia-Romagna Registry (Italy). Eur J Epidemiol 18:773-780

17. Pradat P, Francannet C, Harris JA, et al (2003) The epidemiology of cardiovascular defects, Part I: a study from three large registries of congenital malformations. Pediatr Cardiol 24:195-221

18. Littlewood Teele R, Pease PWV, Rowley RSH (1998) Malrotation in newborns following antenatal diagnosis of intraabdominal cyst. Pediatr Radiol 28:717-721

19. Beddington RSP, Roberston EJ (1999) Axis development and early asymmetry in mammals. Cell 96:195-209

20. Essner JJ, Branford WW, Zhang J, et al (2000) Mesendoderm and left-right brain, heart and gut development are differentially regulated by pitx2 isoforms. Development 127:1081-1093

21. Campione M, Steinbeisser H, Schweickert A, et al (1999) The homeobox gene Pitx2: mediator of asymmetric left-right signaling in vertebrate heart and gut looping. Development 126:12251234

22. Ryan AK, Blumberg B, Rodriguez-Esteban C, et al (1998) Pitx2 determines left-right asymmetry of internal organs in vertebrates. Nature 394:545-551

23. Liu C, Liu W, Lu M-F, et al (2001) Regulation of left-right asymmetry by thresholds of Pitx2c activity. Development 128:2039-2048
24. Dagle JM, Sabel JL, Littig JL, et al (2003) Pitx2c attenuation results in cardiac defects and abnormalities of intestinal orientation in developing Xenopus laevis. Dev Biol 262:268-281

25. Logan M, Pagan-Westphal SM, Smith DM, et al (1998) The transcription factor Pitx2c mediates situs-specific morphologenesis in response to left-right asymmetric signals. Cell 94:307-317

26. Feitz R, Vos A (1997) Malrotation: the post-operative period. J Pediatr Surg 32:1322-1324

27. Murphy FL, Sparnon AL (2006) Long-term complications following intestinal malrotation and the Ladd's procedure: a 15 year review. Pediatr Surg Int 22:326-329

28. Andrassy RJ, Mahour GH (1981) Malrotation of the midgut in infants and children: a 25-year review. Arch Surg 116:158-160

29. Rescorla FJ, Shedd FJ, Grosfeld JL, et al (1990) Anomalies of intestinal rotation in childhood: analysis of 447 cases. Surgery 108:710-715

30. Palanivelu C, Rangarajan M, Shetty AR, et al (2007) Intestinal malrotation with midgut volvulus presenting as acute abdomen in children: value of diagnostic and therapeutic laparoscopy. J Laparoendosc Adv Surg Tech A 17:490-492

31. Borenstein SH, Langer JC (2006) Heterotaxia syndromes and their abdominal manifestations. Curr Opin Pediatr 18:294-297 